\title{
Original Article \\ Open \\ Patient-centred medicine and the broad clinical gaze: Measuring outcomes in paediatric deep brain stimulation
}

\author{
John Gardner \\ Science and Technology Studies Unit, Department of Sociology, University of York, YO10 5DD, UK. \\ E-mail: john.gardner@york.ac.uk
}

\begin{abstract}
Policymakers have argued that patient-centred approaches, which emphasise 'greater patient involvement' and 'comprehensive care', can improve health-care outcomes and lead to a more efficient use of health resources. As a way of anticipating some of the implications of these approaches, this article examines a context that is heavily influenced by the ideals of patient-centred medicine. Drawing on ethnographic research conducted with a multidisciplinary team providing deep brain stimulation to children with movement disorders, this article will illustrate that patientcentred principles can become embedded within particular sociotechnical arrangements involving architectural forms, assessment tools and clinical team structures. These arrangements, it is argued, are implicated in the emergence of a broad clinical gaze: a clinical interest that extends from the shapes and structures of the body, to the subjective thoughts and emotional state of the patient, to elements of the patient's social context and their ability to act within it. The implications of this gaze will be discussed, and this article will suggest that it constitutes a form of disciplinary power that seeks to reaffirm and perpetuate particular ways of being human.
\end{abstract}

BioSocieties (2017) 12, 239-256. doi: 10.1057/biosoc.2016.6; advance online publication, 7 March 2016

Keywords: disciplinary power; neuroscience; chronic illness; architecture; science and technology studies

The online version of this article is available Open Access

\section{Introduction}

Over the last 40 years or so, a considerable body of literature has emerged within the health sciences promoting a patient-centred approach to health care. What exactly constitutes 'patient-centred care' has not always been made clear, but in reviewing this literature, Mead and Bower (2000) argue that it has several key conceptual dimensions. First, patient-centred care broadly champions the biopsychosocial perspective, in which health and illness are viewed as having important social and psychological dimensions. Hence, the biomedical model with its focus on disease process is considered an inadequate means for understanding health. Second, advocates of patient-centred care also emphasise a 'patient-as-a-person' 
approach to health care. Each patient, it is argued, has a unique biography that affects how they make sense of and how they experience illness. If health care is to reduce suffering, then it needs to recognise patients' own agency in managing illness. Third, the patient-centred approach emphasises the importance of sharing power and responsibility. In contrast to the paternalism of the past, doctor-patient interactions should ideally be egalitarian. Patients should be regarded as experts on their illness, and clinicians should be responsive to this expertise. Mead and Bower (2000, p. 1090) note that notions such as 'user involvement' and 'patient empowerment' have often been used to champion this move away from paternalism.

Advocates for a patient-centred approach, then, emphasise the importance of crafting a health-care system that accommodates the 'whole' patient and accounts for their subjective experience, and that provides patients with a space to be active participants in making choices about how to manage their care. This, it is argued, will ultimately lead to improved health-care outcomes. Clinicians will be more responsive to the day-to-day needs of patients, patients will be more satisfied, and this will result in improvements in health and well-being (Coulter, 2002).

The ideals underlying patient-centred care, particularly its emphasis on patient empowerment and accommodating the 'whole' patient, have gained considerable traction among health-care policymakers. Indeed, patient involvement in health care has been described as "the new policy orthodoxy" (Cribb, 2011). In the United Kingdom, for example, the NHS Constitution, which outlines the core principles of the NHS, states that it aims: "to put patients at the heart of everything it does ... NHS services must reflect, and be co-ordinated around the needs and preferences of patients, their families, and their carers ..." (NHS, 2013, p. 3). In this vein, the NHS agency tasked with driving improvement throughout NHS England (NHS Improving Quality) endeavours to facilitate "patient-led improvement to empower and support individuals ..." and "patient-centred best practice to stimulate, learn, share and spread experience of care best practice" (NHSIQ, 2014, p. 5). In addition, initiatives such as NHS Choices - an online health information service - have been established with the aim of facilitating the empowerment of patients, and there have been calls for greater integration of health and social services, and greater cooperation between health professions, to ensure more comprehensive care.

Alongside evidence-based medicine (EBM), 'patient-centredness' may represent one of the major transformative trends within health care in recent times. As May and Mead (1999) have argued, clinical practice has become saturated with patient-centred discourse, as notions such as empowerment, enablement and patient-centredness have become common place in professional vocabulary. There remains, however, a notable gap between this new policy orthodoxy and routine clinical practice. It is, Cribb (2011) suggests, difficult to translate a general set of patient-centred principles into routine clinical practice, and clinicians may express hesitancy because of legitimate dilemmas that can arise from greater patient involvement in some clinical settings. In addition, clinical settings vary significantly according to their particular ethical concerns and sociotechnical infrastructure, meaning that the actual impact of patient-centred care will vary across clinical contexts. What patient-centred clinical practice and care will look like (and how it should look like), will differ from one clinical setting to another, according to the skill sets of clinicians, the availability of particular tools and protocols, and of course, the nature of the condition (that is, chronic or acute) being treated. With this in mind, and with the intention of anticipating some of the social 
consequences of patient-centred approaches in the near future, this article examines routine clinical work in a context that has already been heavily influenced by the principles of patientcenteredness: paediatric neurology. Given the apparent enthusiasm for the ideals of patientcentred care, and given that it advocates a significant readjustment in the nature of clinical work, it warrants careful sociological analysis. Using a specific case study, this article explores how patient-centred care is implicated in reconfiguring patient-clinician interactions, and how it is implicated in reconfiguring the way in which health and illness are understood, measured and managed.

The case study is a multidisciplinary clinical team, the Paediatric Motor Disorder Service (pseudonym) providing deep brain stimulation (DBS) to children with movement disorders. Drawing on ethnographic data (interviews and field notes of observations), we will see that the team inhabits an institutional context that is broadly supportive of patient-centred care; the hospital within which they work was specifically designed to encourage patient-centred care; its multidisciplinary structure is intended to ensure that children and their families receive comprehensive care; and team members deploy tools and protocols that have been developed in disciplines (such as occupational therapy) heavily influenced by the ideals of patient-centred medicine. Using specific examples, this article will explore how patientcenteredness is performed in their clinical work, and in particular, it will explore how they go about measuring the effectiveness of DBS in managing a movement disorder called dystonia. The team has adopted a clinical assessment tool from occupational therapy that, team members argue, captures clinical improvements that patients and their families find meaningful. We will see how the team's use of this tool, along with other features of their clinical context (such as patient-centred architecture and space), are implicated in structuring clinician/patient interactions, and shaping the way in which health and illness are rendered intelligible.

In this article I will suggest that patient-centred care is, in some clinical contexts, characterised by an increasing mobilisation and pooling-together of expertise and tools from different disciplines. As part of this, a wider array of patient attributes are delineated as 'signifiers' of health and illness, and allied health professions are increasingly granted with the authority to speak about, define and delineate disease and its impact upon a patient. One consequence of this is what I will describe as a broadening of the clinical gaze, in which the clinical scrutiny is directed towards various biomedical, psychological and social aspects of the patient. In light of these observations, I will suggest the patient-centred practices can constitute a form of disciplinary power.

\section{Making Sense of Patient-Centred Care}

Sociological studies have emphasised the importance of exploring how ideals of patientcentredness are actually performed in clinical settings (Dubbin et al, 2013; Liberati et al, 2015). Dubbin and colleagues, for example, apply their Bordieuian analytical notion of cultural health capital to examine how human agents' (clinicians and patients) cultural resources, dispositions and interactional styles are implicated in the interactional achievement of patient-centred care. Liberati and colleagues also note the importance of tacit knowledge and dispositions in enabling patient-centred care, but they suggest that it has a material 
dimension: patient-centred care, they argue, is a negotiated achievement involving social practices and material affordances (Liberati et al, 2015).

Following on from these studies, this article is predicated on the assertion that it is important to explore how patient-centredness is performed in practice, and that it is possible to make sense of patient-centred care as being constituted by and perpetuated by various material elements and sociotechnical devices (in addition to human dispositions, and interactional styles). According to this conceptualisation, which reflects the 'post-social turn' championed by STS and Actor-Network Theory in particular (Latour, 2005; Law, 2008), technologies, tools and the built environment reflect the ideals of the humans that engineered them, and thus can (but do not necessarily) actively contribute to the perpetuation and enactment of these ideals. Various technologies have been engineered with the intention of facilitating patientcenteredness in clinical contexts: decision support tools that aim to engage the patient (cf. O'Connor et al, 1999; Thomson et al, 2007); clinical assessment tools that attempt to gauge the wider, psychosocial impact of disease, and patient-friendly spaces within hospitals and clinics. While such elements obviously do not ensure that the ideals of patient-centredness are enacted (as with all technologies they possess a degree of interpretive flexibility (Kline and Pinch, 1999)), they nevertheless represent an important means by which patient-centredness disseminates and becomes embedded in clinical contexts. Studying how such technologies and tools are operationalised, how they are combined and adapted to align with other technologies and other considerations, and examining just what affects they actually bring about (and how they are implicated in power dynamics) is an important task for social scientists.

Two important studies have taken this approach. May and colleagues (May et al, 2006) have explored patient-centred inspired decision-support tools, which, they note, provide something of a systematic, technological solution to the potentially conflicting impulses of patient-centred medicine and EBM. These tools can come in several forms (pamphlets, audio tapes, interactive computer programmes) and they embody aspects of both patient-centred care and EBM: they prompt clinicians to share evidence-based information with patients, and they provide space for some patient input, thus encouraging patients to make "evidencebased choices" (May et al, 2006, p. 1026). In effect, May and colleagues argue, these tools structure and manage patient/clinician interactions, and they mechanically elicit patient preferences according to pre-set criteria, rather than, say, accommodating the heterogeneity of patient subjective experience. Similarly, Armstrong et al (2007) have explored another technology that has perpetuated patient-centredness: health-related Quality of Life (QoL) instruments. The emergence of these instruments, they argue, reflects a transformation in medical thinking. In the past, disease and its symptoms were anchored within the fleshy body of the patient, and it was the body that was the focus of the clinician's normative gaze. The emergence and dissemination of QoL instruments both reflected and crystallised a clinical interest in the distal effects of disease: its impact on day-to-day living (such as impaired mobility or disrupted personal relationships), and its impact on a patient's overall sense of well-being. In effect, Armstrong et al argue, the symptoms of disease were detached from the fleshy body and relocated within the social and psychological domain, and disease, therefore, has been rendered intelligible as having a subjective dimension as an experienced 'illness'. The widespread dissemination and usage of QoL instruments in clinical contexts has firmly consolidated this clinical interest in the social and psychological aspects of disease. 
By exploring specific technologies, both these studies have drawn attention to the biopolitical consequences of patient-centred clinical work. From this perspective, the patientcentred approaches are constituted by a machinery of technologies implicated in the governing of clinical interactions and in the subjection of patients and aspects of their lifeworld to clinical scrutiny. Similarly, this article will also explore the consequences of patient-centred clinical work by focusing on the use of a patient-centred technology: the Assessment of Motor and Process Skills (AMPS). This article will illustrate how this clinical assessment tool is operationalised within the Paediatric Motor Disorder Service (PMDS) as a means of measuring the effectiveness of DBS, and in the process, we will see that it provides a standardised framework for conducting assessments, in which particular patient preferences are elicited, and in which the attention of clinicians is directed towards the day-to-day domestic life of the patient. It provides a highly configured opening for patient participation and, this article will argue, it is implicated in facilitating a broad clinical gaze.

The broad clinical gaze can be defined as a clinical interest that extends from the shapes and structures of the body, to the subjective thoughts and emotional state of the patient, to elements of the patient's social context and their ability to act within it. The notion derives from Foucault's (1963/2003) characterisation of the medical gaze: a mode of perceiving disease, and rendering disease intelligible, according to empirically observable, biological phenomena within the fleshy, concrete spaces of the body. The medical gaze therefore perpetuates the biomedical model of disease, and it is also normative: it is implicated in practices aimed at identifying and correcting biomedical aberrations. The broad clinical gaze is also normative, but as Armstrong and colleagues illustrate with QoL measures (Armstrong et al, 2007), it is no longer tethered to the disease process and its direct effects on the body: rather, a range of other patient attributes are foregrounded, or 'brought to light', and are delineated as signifiers of disease. Within the PMDS the clinical gaze is directed towards biomedical and psychological aspects of the patient, their relationship with peers and family, and as we will see with team's use of the AMPS, the patient's domestic body technique: their ability to efficiently use their body to perform culturally mediated domestic tasks. One consequence of this is that these attributes may be subject to corrective measures.

Tools such as the AMPS are of course embedded within a wider infrastructure, and this article will highlight other institutional factors that encourage patient-centred modes of clinical work and the broad clinical gaze. First, the multidisciplinary composition of the team itself both reflects patient-centred ideals, and perpetuates patient-centred modes of clinical work. Bourret (2005) has argued that the emergence of forms of molecular clinical practices in cancer genetics was predicated on the formation of particular multidisciplinary team structures involving biology, oncology and other clinical disciplines. These novel clinical practices (which constitute what could be called a molecular gaze) emerged from the pooling of resources and expertise from different disciplines. Using the PMDS as a case study this article will illustrate how, in a similar fashion, the pooling of resources and expertise from various disciplines, particularly the allied health professions, can result in the emergence of a broad clinical gaze.

Second, space and architecture also contribute to the perpetuation of patient-centred clinical practices and the broadening of the clinical gaze. Various scholars have explored the relationship between medical knowledge, hospital design and biomedical practice (cf. Prior, 1988; Keating and Cambrosio, 2003; Adams, 2008). Prior (1988) has argued, for example, that the spaces that 
constitute the built environment of a hospital should be seen as a social product as they reflect particular beliefs about the patient and the nature of disease (as well as reflecting engineering and aesthetic considerations). In addition, such spaces are not merely inert backdrops to human interaction. Their symbolic and material dimensions guide, restrict and facilitate particular activities within. Drawing on the work of Foucault, Prior argues that hospital architecture configures particular forms of clinical work, and thus participates in perpetuating biopolitical practices of surveillance and discipline. As this article will illustrate, the hospital within which the PMDS team is based was specifically designed to encourage a comprehensive patient-centred service. It contains spaces that afford particular forms of clinical work in which various 'nonbiomedical' attributes of the patient are foregrounded and delineated as useful signifiers of disease. It permits, as we will see, the construction of a domestic assessment space in which the AMPS is conducted, and domestic body technique is examined.

The PMDS illustrates how it is that 'patient-centredness' can become embedded within sociotechnical arrangements, and it provides an opportunity to see how it is that such arrangements are implicated in reconfiguring clinician/patient interactions, and the way in which illness is understood, measured and managed. The sociotechnical arrangement outlined here is specific to paediatric neurology. Nevertheless, it can serve as a useful reference for anticipating some of the implications of the patient-centred approaches more generally, as particular architectural forms, inter- and multidisciplinary arrangements and tools are adopted and implemented in other health-care settings.

\section{Methods}

The data for this article were collected during an ethnographic study of the PMDS team, based at a children's hospital in the United Kingdom. The study was conducted as part of a Wellcome Trust-funded PhD project (Gardner, 2014), and indeed this article reports on the key findings of the project as a whole. The study took place over a period of 12 months and included mixed qualitative methods: observations of team meetings $(n=31)$; in-depth, semistructured interviews with members of the clinical team and a previous clinical director of the hospital $(n=12)$; and observations of interactions $(n=6)$ involving team members, patients and the patient's supporting family members. The objective of this fieldwork was to explore what Morlacchi and Nelson (2011) refer to as 'learning in practice': how it is that team members learned to integrate the DBS technology into an actual, day-to-day therapy for dystonia. The interviews, therefore, were used to explore team members' perceptions of the challenges associated with the integration of DBS. These were audio-recorded, transcribed and coded according to emerging themes pertaining to key challenges. The observations were used to examine how these challenges actually manifested and were managed in day-to-day clinical practice. Observations were documented in field notes. Importantly, during the observations I sought to describe the language and phrasing employed by participants, and also the material context in which the interactions took place. This is on the assumption that human activity is not only culturally and historically situated, but materially situated (Mol, 1999). Ethical approval was obtained from a relevant NHS Research Ethics Committee. Informed consent was obtained from all PMDS team members and all patients over 16 years of age, and their supporting family members involved in observations. For patients under 
16 years of age, their assent was obtained, and informed consent for their involvement was obtained from their guardian.

\section{The PMDS Team and the Hospital}

The PMDS is one of the few teams worldwide providing DBS specifically to children with a movement disorder called dystonia. It is a multidisciplinary team that includes three neurologists (including a research fellow), a specialist nurse, two physiotherapists, an occupational therapist (OT), a speech and language therapist, and a clinical psychologist. The team was structured in this way to provide 'comprehensive' care, and it reflects a biopsychosocial understanding neurological disease, its effects and the role of DBS.

Dystonia is caused by abnormal neural activity in the basal ganglia of the brain. This causes sustained or intermittent muscular contractions, often resulting in painful and crippling body postures. Within the PMDS, dystonia is also understood in terms of its impact on a patient's engagement with their surrounding world: how it affects, in other words, their functionality. In severe cases of dystonia, patients become 'locked' in their body, and children who have dystonia from a young age are unable to acquire many of the skills that usually result from constant engagement with their surrounding world. DBS, which involves using a pacemakerlike device to deliver constant electrical stimulation to the brain, does not cure dystonia, but it can reduce dystonic movements significantly, and this enables therapists to work with patients to obtain useful functional skills. As the research fellow stated:

DBS unlocks dystonia so that the therapists can get in and get you the functional recovery ... It allows you to get some more intensive therapy.

(Interview)

The composition of the team reflects this emphasis on functional recovery: the psychologist and each of the therapists are responsible for evaluating and reporting upon a particular aspect of the disorder's impact upon patients' day-to-day lives.

This emphasis on functionality, and the various roles of each of the team members, is illustrated during the team's weekly meetings. Team meetings provide an opportunity for team members to provide updates based on their formal assessments with patients and their time spent with families, and they provide a space to engage in a collective clinical decision making. They also provide an opportunity to glimpse the clinical gaze of the team as a whole; the way in which the team discursively renders patients (and their illnesses) intelligible by referring to and foregrounding a broad range of elements. During team meetings that were observed as part of this project, each team member tended to draw on their own disciplinary-based understanding of a patient when contributing to the discussion. The neurologist tended to use biomedical frames of reference when describing patients, such as DBS stimulation parameters, medication regimes and brain structures. The psychologist would refer to cognitive ability, mood and selfperception, for example: "he is lonely and has poor self-esteem. He has never had suicidal ideation ...". The speech and language therapist would refer to problems with swallowing and eating, and speech and verbal communications skills: "she speaks very quickly - once she starts speaking she has trouble stopping"; and the OT would report on patients' ability to perform particular domestic and occupational tasks (as we explore in further detail below). Thus, during 
discussions, each team member is acting as a 'spokesperson' (Latour, 2005, p. 31); an agent that speaks with authority about, and thus delineates, a particular aspect of the patient's illness. As each team member contributed their discipline-specific input, a composite, broad picture of the patient and their illness would emerge, in which various biomedical, psychological and social referents are used to make sense of the illness and inform decision making. Patients and their illnesses are, in other words, discursively enacted as having biomedical dimensions, as well as social and psychological dimensions.

In addition to this, team members would also report upon and discuss patients' domestic environment. The following team meeting extract provides an example of this. Here, one of the neurologists is describing the home life of Ioannis, a 5-year-old child with severe dystonia:

We need [the psychologist] to have a discussion with the family. The family is in distress. Iaonnis has been waking up early in the morning, screaming ... and then in the evenings they are having trouble getting him into the bath. I think there is a large behavioural component to what is going on. I think he is intensely jealous of his older brother who is running around, playing ... It is difficult for the family ... We will bring him in to the ward for four of five days ...

In this extract, the implications of the illness on Iaonnis' state of mind on his relationship with his sibling and on his family are foregrounded. Accounts of this type were common and team members would often discuss a patient's relationship with their siblings and parents, peers, and their schooling. In effect, then, many aspects of the patient, including aspects of their family life, were subjected to clinical scrutiny, or a broad clinical gaze: a clinical interest that extends from the inner concrete shapes and forms of the body, to the subjective thoughts and emotions of patients, to the internal dynamics of domestic life.

This collectively enacted gaze is normative. Patients were subjected to various biomedical, psychological and social norms, and courses of action would be considered in an attempt to align patients with these norms. The following extract from a team meeting discussion involving the OT and a neurologist (Neur) illustrates this.

OT: He has been out of school for three months. He has temper tantrums. His mum seems to be handling things very well ...

Neur: We can increase [medication to help with mood]. If things are difficult at school, we can contact the school and talk to them.

Here, the patient's mood is deemed in need of correction and may warrant making contact with support services at his school. Similarly, in the following example the psychologist, while commenting on a patient's social skills, proposes to help her family reduce her levels of anxiety.

I saw her today for a cognitive assessment. She was showing improvement. She has more confidence; she is making more friends ... Her main problem seems to be anxiety ... I will give the family a couple of sessions on how to manage her anxiety.

These examples thus provide a glimpse into the potential normative implications of the broad clinical gaze. In the PMDS it is characterised by multidisciplinary team practices that render an individual intelligible by delineating and foregrounding various aberrations, and it entails the attempted correction of particular aberrations that are deemed to be 'abnormal'. 
Patients are subjected to this broad clinical gaze throughout their time with the PMDS. Before the DBS system is implanted, patients undergo a regime of assessments, many of which will be repeated at regular intervals (every 6 months for the first 2 years, and then every 12 months) after the system has been implanted. These reflect the multidisciplinary nature of the team and include neurological examinations and central motor conduction time tests; speech and verbal fluency assessments; assessments of cognitive function and mood; assessments of pain; assessments of gross motor function; and, as we shall explore in more detail further on, assessments of a patient's ability to perform domestic tasks. During each of these assessments the clinical gaze is directed towards specific features of the patient, and collectively they produce a broad picture of the patient and the impact of their movement disorder. The assessments are standardised, and importantly for the team they generate data that can subsequently be used to determine the effectiveness of DBS.

These various forms of assessment each require particular types of tools and spaces. In this regard, the team takes advantage of the newly built children's hospital within which they work, which was specifically designed to encourage multidisciplinary, patient-centred care. Children were consulted on aspects of the design (which has influenced the selection of furniture and the design of corridors), and focus groups involving nurses, physiotherapists, OTs and doctors were consulted on functional aspects of the building (ex-clinical director, Interview). Consequently, the hospital contains an open-plan office area, in which clinical teams such as the PMDS are encouraged to sit together as a group. The hospital contains a gymnasium, a school for patients and their siblings to attend, and each ward contains a family-friendly space containing a kitchenette, a play area and table and chairs for dinning. Such features reflect a belief that the hospital is not simply treating and managing disease processes in individual children; rather, it is dealing with families and the psychosocial aspects of disease. As the ex-clinical director (who was clinical director at the time the hospital was designed and built) stated:

You always have to remember that a child is part of a family and anything that happens to the child has ramifications for siblings and parents ... The decision was made when we were setting up that if were really going to help the children who are in hospital, we need to think holistically about the whole family.

(Interview)

The hospital design, then, is the consequence of collaborations involving various health professionals with some patient input, and the resulting built environment reflects a clinical concern for patients' wider social context. Importantly, it provides spaces for allied health professionals to engage in particular forms of clinical work, during which the disease and its impact are rendered intelligible. The gymnasium is used by the PMDS physiotherapists to assess the impact of dystonia on patients' gross motor function, and the kitchenette, as we will see, provides the space and tools required for the OT to conduct the AMPS. In effect, the hospital design provides affordances for the type of interactional patient-centred practices that constitute the broad clinical gaze.

\section{The Assessment of Motor and Process Skills (AMPS)}

One of the key assessments conducted with patients is the AMPS. In this section, after providing some background on the AMPS, we will see how it is operationalised within the 
PMDS, using two assessment interactions as examples. These will enable us to see how it is that architecture and space are implicated in patient-centred clinical work and the broad clinical gaze.

The AMPS was developed in the early 1990s by the OT Anne Fisher, and it is designed for OTs to evaluate the impact of interventions on clients with any form or degree of disability. In accordance with the underlying patient-centred core concept of occupational therapy, it is intended to evaluate clients' capacity to engage in specific occupational and domestic activities that clients themselves feel are important (Fisher, 2001). Generally the AMPS is carried out with clients in the community in their domestic setting. It is, nonetheless, designed to be a highly standardized, carefully controlled means of evaluation. In order to maintain a high degree of uniformity, for example, clinicians who wish to use the AMPS must gain approval from, and be calibrated by, a regulatory agency known as the Center for Innovative OT Solutions.

Conducting the AMPS entails following the pre-set format provided by the AMPS manual. To an important degree, the manual structures the assessment interaction by designating the activities to be carried out by those involved, and in doing this, it also provides a highly configured space for patient involvement. It constitutes, in other words, a script (Berg, 1998) that prescribes the roles of participants, and in doing so, it directs the clinical gaze towards specific patient attributes. Before the AMPS is conducted the client must choose several occupational or domestic activities that they feel are important to them (such as brushing teeth, preparing a bowl of cereal, self-dressing) from a list of 120 standardised 'activities of daily living (ADL)' provided in the AMPS manual. For each ADL, the manual provides a description of how the task is to be performed during the assessment, and it describes how that tasked should be graded by the OT. The therapist will examine and grade (from 1 to 4 ) 36 specific skill items that constitute all of the ADL. These include 16 motor skill items (such as walking, reaching, gripping objects) and 20 process skill items (such as choosing objects, pacing), all of which are defined by the AMPS manual. Once all items have been scored, the therapist enters the scores into an AMPS software programme, which subsequently computes an overall score for that particular client. During the assessment, then, patients are performing tasks that they feel are important to them, but they are doing so in a manner described by the AMPS manual, and they are being scored according to criteria set-out in that manual.

\section{A domestic assemblage}

In many occupational therapy contexts the application of the AMPS takes place in the client's home. This provides the OT with an opportunity to observe a client as they attempt to perform tasks that are important to them, in the environment in which they would usually perform these tasks. As the manual states:

[OTs] need to observe and evaluate ADL task performances in natural spaces: bedrooms, family or living rooms, kitchens, gardens - ones like those where the client typically would be performing ADL tasks.

(Fisher and Jones, 2010, pp. 4-5)

In the context of the PMDS, where patients and their families may spend several days undergoing an assortment of assessments, it is necessary to recreate these 'natural' spaces within the hospital. The kitchen facilities, the play area with toys, and the table and chairs for 
dining provide the OTs with a well-equipped setting to carry out AMPS assessments. In it is in one of the spaces that the OT conducts an assessment with Carl, a 16-year-old male patient with secondary dystonia. We will focus here on one of the tasks chosen by Carl: washing the dishes. Carl would like to attend university in a few years, and this would require him to live away from his mother and clean up after himself.

In order to conduct the assessment, the OT carefully prepares a layout of objects in the hospital kitchenette. This spatial layout, as we will see, enables particular information to be extracted from the patient, in much the same way that the careful arrangement of a laboratory assemblage enables information to be obtained from an entity of interest (Latour and Woolgar, 1979; Mol, 2002). Particular interactions can take place within, shielded from 'noise', in which the perceptual capacities of the clinician - the gaze - can be attuned to specific aspects of the patient (Gardner and Williams, 2015). The assemblage created by the OT is standardized according to the AMPS manual: in describing how each task is to be performed by the patient, it also designates the types of objects to be included and how they should be arranged within space. 'Washing the dishes' corresponds to ADL task J-2 in the manual, which states that the task must be performed as follows:

The client is expected to wash and rinse 10 to 15 dishes ... Rinsing soap suds off the dishes is expected ... Appropriate dishes include an assortment of plates, glasses, silverware, small pans and related utensils. [After the dishes have been cleaned] the client is to drain water from the sink, wipe the counters dry, wring out the dishrag or sponge.

(Fisher, 2003, p. 100)

Having checked the manual she has with her, the OT arranges the kitchenette so the task can be carried out correctly. She and the assistant have brought with them a box of dirty dishes from the staff offices on the upper levels. The dishes (bowls, plates and cutlery) are piled on one side of the sink and the dish rack is placed on the other. The OT checks that there is dishwashing liquid, a sponge and a tea towel in the cupboard below the sink. In effect, the home is brought into the clinic. The assemblage created by the OT is intended to mimic the spatial layout of objects in an 'average' domestic kitchen. The OT then familiarises Carl with the ADL task:

OT: For this [task], you are going to rinse and wash the dishes, stack them, dry them, and then put them away, and then you are going to wipe the bench and the sink clean. Got it? Now, you will put the clean plates here and the clean bowls here [in the dish rack] and we will put the cups and utensils over here [next to the dish rack]. You can also put the sponge and the tea towel where they should go when you have finished. Happy?

Carl: Yeah.

Within this ordered space, Carl is encouraged to interact with the other elements of the domestic assemblage in much the same way as an 'average' individual would at their home. After receiving his instructions, he stands up and walks to the bench, sighs loudly and begins to get on with washing the dishes and putting them on the rack one by one. The OT watches closely and takes notes with a pad and pen, and the therapy assistant stands nearby recording 
Carl with a small handheld camcorder. Once all the cleaned dishes have been placed on the rack, Carl rinses the sponge and wipes the bench and sink. Carl seems tired and moves slowly, and his body shakes because of his movement disorder, but he appears to complete the task without much trouble.

At the end, the physiotherapist has produced a video recording and the OT has created a series of notes. The OT will subsequently use these to closely scrutinise Carl's performance and provide scores for each of the 36 motor and process skill items, thus enabling an overall sore to be calculated.

\section{An assessment of body technique}

For each of the motor and process items, the OT must assign a number between one and four, where four equates to "performs skill item readily and consistently", and one "equates to severe deficit or inability to perform skill item" (Fisher, 2003). In order to illustrate the scoring process and its implications, we will follow the OT as she uses a video recording to provide scores for a patient who had earlier performed the preparing 'Cold cereal and beverage' ADL task (task C-1). The patient, William, is 7 years old and has primary dystonia. This performance was one of several conducted as part of a post-surgical review: William has had the DBS system implanted for 1 year.

Before scoring each skill item the OT watched the video recording of the performance from start to finish. It begins with the OT giving William his instructions: He is to get the milk from the fridge, a glass from the top right cupboard and use the jug of water that has been provided. The OT shows William where these various objects are. William begins by grabbing a bowl from the cupboard and placing it on the bench. William then performs the task without any obvious difficulty.

The OT then begins scoring each of the 36 motor and process skill items that constitute William's performance, one by one. The scoring process involves an examination of the way in which William uses his body to negotiate and interact with other objects within the domestic assemblage. In effect, each skill item draws the clinical gaze to a specific aspect of this interaction.

Several motor and process skills such as 'Aligns' and 'Positions' relate to the position of the body relative to other objects within the domestic assemblage. Importantly, body alignment and position are rated according to the degree of functionality: a higher score is achieved if the body is orientated in such a way that the ADL task can be completed efficiently. If the body alignment results in "unacceptable delay, unacceptable effort" or "task breakdown", it is scored as a 1 (Fisher, 2003, p. 189). Here is an example from the scoring of William's performance (as the OT scores each item, she explains her reasoning to me):

OT: The second [skill item] is 'Aligns' - this is also a 4. He had no problem positioning himself to do the task. [Next skill item is] 'Positions' - this is something you would notice when their elbow is way up in the air while they are trying to pour something for instance. There was a bit of that with William. It kind of looks a bit awkward. Let's give him a 2 .

Some motor and process skill items pertain to specific interactions between the body and other objects within the domestic assemblage. For example, 'Grips' refers to the ability of the patient to grasp an object (such as a plate or a pan) or to open containers, 'Lifts' refers to the patient's ability to lift (and not slide) an object from one position to another and 'Manipulates' refers to 
dexterity or in-hand manipulation of task objects. Again, body-object interactions are rated according to their functionality:

OT: 'Grips' - there was no grip slip - 4. Even if you see a quick grip slip then you give him a 2, but there was nothing like that here ... 'Lifts' - If you slide an object rather than lift it, then you score it down. Did he do it? I think he slid the jug of water across the bench. This is where the video is useful [the OT consults the recording]. He did! He gets a 2 ... 'Manipulates' - there was some fumbling with the cereal when he was trying to get it out of the box. He gets a 2 for this.

Body-object interactions are also graded according to their appropriateness. The 'Uses' skill item refers to whether or not the correct object has been selected for a task: using "a plate as a plate, knife as a knife" (Fisher, 2003, p. 207). Here, then, the scoring process reflects social conventions regarding the use of particular objects. This is illustrated in the following extract, where I am asked for my opinion on the appropriateness of an interaction between the body (William's hand) and an object a (bowl of cereal):

OT: 'Uses' - this is about hygiene. For instance, does a stroke victim use a toothbrush to brush their hair? There is an issue with William's hands in the cereal. What do you think? JG: For me, seeing him use his hand to adjust the dry cereal in the bowl looked quite normal ... But when he used his hand to move the milky cereal, that was different. That seemed unhygienic.

OT: Yes, I agree. He gets a 2.

The fluidity of body movements while performing the task is also graded. 'Flows' pertains to the 'fluid quality' of arm and hand movement. Smooth movement scores highly, whereas movement that is disrupted, perhaps from "tremor, stiffness, increased tone" is scored down (Fisher, 2003, p. 196):

OT: 'Flows' - This is hard, I will look [at the manual]. I don't think the spillage was due to "marked spillage due to tremors", which would qualify as a 1 in the manual. I think it was due to orientation. So, I will give him a 2.

Scoring skill items also involves differentiating 'erroneous' movements from functional movements. Movements that are not directed towards the completion of the task, or hinder the completion of the task, may result in a lower score. For example, a "wobbly while walking or interacting with task objects" will result on low score of 'Stability' and 'Transports'. A client's inappropriate persistence with a task will also be designated as erroneous movement:

OT: 'Terminates' - William gets a 1 for this. He continued to pour milk into the bowl of cereal, even when it was full to the top. He did it twice!

Other skill items pertain to the client's control over their body while moving and interacting with other objects. For example:

OT: 'Calibrates' - Here we are grading his movement and how he adjusts the force of his movement depending on the object. Does he use too much force? Not enough? Well, you can see how much milk he spilled. He gets a 1 . 
The temporal dimension of the performance is also rated. 'Sequences', for example, is scored highly if various steps are performed "in an effective order for efficient use of time" (Fisher, 2003, p. 214). Similarly, 'Paces' pertains to the overall rate of the task:

OT: 'Paces' - I think he is slow. He is consistent, but he is slow. So, he gets a 2.

By the time all 36 items have been scored, the OT has scrutinised: the positioning of the William's body within the domestic assemblage, the interactions between the body and other elements within the assemblage, body movement and fluidity of movement, and the rate at which sequences of body movements occur. Importantly, as William's example illustrates, these various aspects of the performance are rated according to their functionality and efficiency. During the assessment, then, the clinical gaze of the clinician is directed towards the patient's ability to use the body as an efficient implement: an implement for carrying out particular domestic tasks in a culturally permissible manner. In other words, the AMPS directs the clinical gaze of the clinician towards the patient's domestic body technique. These techniques involve a specific series of bodily movements and forms; they are social in that they are learned within cultural contexts; and they are efficient in that they serve a specific function.

Within the PMDS, these features of body technique are scrutinised according to particular norms. Indeed, the AMPS enables the patient's proficiency in domestic body techniques to be quantified and compared with others. Once the OT has decided upon scores for all 36 items, she enters them into the AMPS software, along with the ADL task code and the age of the patient. The programme combines these details with scores from several of William's other ADL performances, enabling the OT to compare these with those of the 'average, able-bodied' 7-year-old child, and with those of William's pre-surgical baseline assessment: The OT notes that William's scores are within the normal range of an average 7 year old, despite scoring poorly on several skill items. And, she states that, when compared with his pre-DBS scores, "his motor skills have improved significantly, although his process skills are largely unchanged". It is according to such comparisons of body technique proficiency that the AMPS enables the impact of the motor disorder, and the effectiveness of DBS as therapy for managing dystonia, to be assessed for each patient.

\section{Discussion}

In the Birth of the Clinic (1963/2003), Foucault describes the emergence of what he referred to as the clinical gaze of modern medicine. This gaze, he argues, seeks to delineate and define the body in its concrete shape and form, and its emergence at the end of the eighteenth century signalled the birth of the biomedical model of disease and the body. With the medical gaze, the 'truth' of disease was to be found in the body and the lesion, neither of which were accessible to the patient without the interpretation of the clinician (Armstrong, 1997). The clinicians were thus endowed to speak with authority about the disease; they became its 'spokesperson', while the patient, along with their social circumstances are largely elided or are considered irrelevant to clinical decision making. For Jewson (1976) this new patient-doctor relationship was crystallised in the emergence of the modern hospital. Patients were dislocated from their personal domestic contexts and arranged in uniform clinical spaces; spaces that facilitated clinical work aimed at deciphering and delineating the body and the lesion. Within the patient-centred practices of the PMDS, something 
different to the Foucault's characterisation of the clinical gaze is in operation. Biomedical considerations are certainly important, and without a doubt such considerations influence a great deal of decision making, but other aspects of the patient are also foregrounded. The team conducts various assessments with patients, and as we saw during team meetings, a broad range of elements relating to mood, speech capacity, domestic life and so on are delineated and discussed during group decision making. Such elements and anomalies are, so to speak, 'brought to light' and used as signifiers of the disease and its impact upon the patient. In light of this I have suggested that the PMDS casts a broad clinical gaze over their patients: a gaze that extends from the inner concrete shapes and forms of the body, to the subjective thoughts and emotions of their patients, and to the internal dynamics of domestic life. As with the medical gaze described by Foucault, the broad clinical gaze of the PMDS is reflected in, and constituted by, architectural forms and particular technologies and tools. The example of the teams' use of the AMPS tool, which entails a careful construction of a domestic space, illustrates how the gaze is directed towards domestic body technique. In effect (and in contrast to Jewson's point), the patient has been relocated within the domestic sphere.

In their ethnographic study, Dubbin et al (2013) note that patient-centred practices performed tend to be characterised by an asymmetrical power dynamic. Similarly, we can see that this is a feature of the PMDS' broad clinical gaze. Physiotherapists, a speech and language therapists, a psychologist and - as we have seen in some detail here - an OT, are each endowed with authority to speak about and delineate some aspect of the disease and its impact. In this particular case study the broadening of the clinical gaze has entailed the enrolment of a broader range of experts into a project aimed at assessing and delineating the patient and their illness, which to an extent restricts the patient's capacity to act as a spokesperson. The AMPS is a good example of this. Patients, as we saw with the example of Carl, are provided some scope for decision making: they can identify those tasks of daily living that are important to them, and that will enable a point of reference for measuring the effectiveness of DBS. But they must choose from a list of provided tasks, and they must perform the task in the manner described by the AMPS manual, within a space that has been standardised. The interaction is to a large degree scripted, and it is designed to elicit certain pre-set preferences from patients. In a manner synonymous with the patient-centred-inspired decision-making aids examined by May et al (2006), these tools mechanically draw data from the patient so that certain quantifiable elements are foregrounded and 'stand-in' for the patient's subjective experience of illness. Like the medical gaze described by Foucault (2003), the broad clinical gaze of the PMDS renders the patient as an object of knowledge by obscuring 'messy' and cumbersome personal detail.

And, like the medical gaze described by Foucault, there are normative consequences for those subjected to the broad clinical gaze of the PMDS. Patients are, in effect, subject to a broad surveillance where they are inevitably compared with various norms and where clinical action is undertaken with the intention of upholding such norms. We can see here, then, how the emergence of the broad clinical gaze, by blurring the distinction between the clinic and the domestic, private world of the individual, may be implicated in the perpetuation of certain modes of existence and modes of living within the world. While the degree to which patients themselves arrogate this mode of understanding and incorporate it into practices of the self has not been explored in this article, we can see how patient-centred medicine may entail a form of disciplinary power. It is constituted by a sociotechnical infrastructure (architecture, tools, team structures, frames of reference) in which patients are described, assessed and 
graded according to specific biopsychosocial traits. Consequently they become discernible, and potentially malleable, according to such traits. In effect, the broad clinical gaze constitutes the individual as a treatable biopsychosocial entity and it constitutes the biopsychosocial individual' as a legitimate way of being human (Rose, 2007).

The PMDS is no doubt unique in the degree to which it has been influenced by the ideals of patient-centred care. However, as an 'early adopter' of a patient-centred approach it does provide a useful point of reference for anticipating the implications of adopting patient-centred care in other contexts, particularly those that, like the PMDS, are involved in managing chronic illness. It enables us to see the how the deployment of patient-centred sociotechnical arrangements (multidisciplinary team structures, architectural forms, technologies and protocols) can have particular effects that may or may not be desirable in specific settings. As part of a move towards patient-centred health-care systems, there have been calls for the greater integration of social and health services within the United Kingdom (NHSE, 2014). Given the picture provided by the PMDS, the initiative may be seen as the propagation of a disciplinary regime; a regime constituted by sociotechnical arrangements that traverse clinical and domestic spaces, and that constitutes people as malleable biopsychosocial citizens.

\section{Acknowledgements}

This article presents the key findings of a PhD project (Gardner, 2014) supervised by Clare Williams and Steven Wainwright. The author is extremely grateful to them for their assistance and direction. The author also thanks the anonymous reviewers of this article, and especially Andrew Webster for his valuable comments on earlier drafts. This project was funded by the Wellcome Trust (Wellcome Trust Biomedical Ethics Strategic Award 086034).

The study upon which this manuscript is based was approved by the appropriate NHS Research Ethics Committee and the ethics committee of the author's host institution at that time. The author declares that there is no conflict of interest.

\section{About the Author}

John Gardner is a postdoctoral researcher at the University of York. His research interests are the dynamics of innovation and social change and the relationship between technology, the body and sociality.

\section{References}

Adams, A. (2008) Medicine by Design: The Architect and the Modern Hospital, 1893-1943. London: University of Minnesota Press.

Armstrong, D. (1997) An Outline of Sociology as Applied to Medicine, 4th edn. Oxford: Butterworth-Heinemann.

Armstrong, D., Lilford, R., Ogden, J. and Wessely, S. (2007) Health-related quality of life and the transformation of symptoms. Sociol Health Illn 29(4): 570-583.

Berg, M. (1998) Order(s) and disorder(s): Of protocols and medical practices. In: M. Berg and A. Mol (eds.) Differences in Medicine: Unraveling Practices, Techniques and Bodies. Durham, NC: Duke University Press, pp. 227-246. 
Bourret, P. (2005) BRCA patients and clinical collectives: New configurations of action in cancer genetics practices. Social Studies of Science 35(1): 41-68.

Coulter, A. (2002) After Bristol: Putting patients at the centre. BMJ: British Medical Journal 324(7338): 648-651.

Cribb, A. (2011) Involvement, Shared Decision-Making and Medicines. London: Royal Pharmaceutical Society.

Dubbin, L.A., Chang, J.S. and Shim, J.K. (2013) Cultural health capital and the interactional dynamics of patient-centered care. Social Science \& Medicine 93(September): 113-120.

Fisher, A. (2003) Assessment of Motor and Process Skills, 6th edn. Fort Collins, CO: Three Star Press, Incorporated.

Fisher, A.G. (2001) Assessment of Motor and Process Skills: User Manual. Fort Collins, CO: Three Star Press, Incorporated.

Fisher, A.G. and Jones, K.B. (2010) Assessment of Motor and Process Skills. Vol. 1: Development, Standardization, and Administration Manual, 7th edn. Fort Collins, CO: Three Star Press.

Foucault, M. (1963/2003) The Birth of the Clinic. London: Routledge Classics.

Gardner, J. (2014) A sociology of medical innovation: Deep brain stimulation and the treatment of children with dystonia. A thesis submitted to Brunel University London for the degree of Doctor of Philosophy (PhD), http://bura.brunel.ac.uk/bitstream/2438/8714/1/FulltextThesis.pdf.

Gardner, J. and Williams, C. (2015) Corporal diagnostic work and diagnostic spaces: Clinicians' use of space and bodies during diagnosis. Sociology of Health and Illness 37(5): 765-781.

Jewson, N.D. (1976) The disappearance of the sick-man from medical cosmology, 1770-1870. Sociology 10(2): 225-244.

Keating, P. and Cambrosio, A. (2003) Biomedical Platforms: Realigning the Normal and the Pathological in Late-Twentieth-Century Medicine. Cambridge, MA: MIT Press.

Kline, R. and Pinch, T. (1999) The social construction of technology. In: D. Mackenzie and J. Wajcman (eds.) The Social Shaping of Technology. Philadelphia, PA: Open University Press, pp. 113-115.

Latour, B. (2005) Reassembling the Social: An Introduction to Actor-Network Theory. Oxford: Oxford University Press.

Latour, B. and Woolgar, S. (1979) Laboratory life: The Construction of Scientific Facts. Los Angeles, CA: Sage.

Law, J. (2008) Actor-network theory and material semiotics. In: B. Turner (ed.) The New Blackwell Companion to Social Theory. Oxford: Blackwell, pp. 141-158.

Liberati, E.G., Gorli, M., Moja, L., Galuppo, L., Ripamonti, S. and Scaratti, G. (2015) Exploring the practice of patient centered care: The role of ethnography and reflexivity. Social Science \& Medicine 133(May): 45-52.

May, C. and Mead, N. (1999) Patient-centredness: A history. In: C. Dowrick and L. Frith (eds.) General Practice and Ethics. London: Routledge, pp. 76-91.

May, C., Rapley, T., Moreira, T., Finch, T. and Heaven, B. (2006) Technogovernance: Evidence, subjectivity, and the clinical encounter in primary care medicine. Social Science \& Medicine 62(4): 1022-1030.

Mead, N. and Bower, P. (2000) Patient-centredness: A conceptual framework and review of the empirical literature. Social Science \& Medicine 51(7): 1087-1110.

Mol, A. (1999) Ontological politics. A word and some questions. The Sociological Review 47(S1): 74-89.

Mol, A. (2002) The Body Multiple: Ontology in Medical Practice. London: Duke University Press.

Morlacchi, P. and Nelson, R.R. (2011) How medical practice evolves: Learning to treat failing hearts with an implantable device. Research Policy 40(4): 511-525.

NHS (2013) The NHS Constitution: The NHS Belongs to Us All. London: Crown.

NHSE (2014) NHS England Chief offers local government leaders radical new health and social care integration option [Press release], http://www.england.nhs.uk/2014/07/09/ipc-prog/, accessed 12 February 2015.

NHSIQ (2014) An Introduction to NHS Improving Quality. London: NHS Improving Qaility.

O'Connor, A.M. et al (1999) Decision aids for patients facing health treatment or screening decisions: Systematic review. British Medical Journal 319(7212): 731-734.

Prior, L. (1988) The architecture of the hospital: A study of spatial organization and medical knowledge. The British Journal of Sociology 39(1): 86-113.

Rose, N. (2007) The Politics of Life Itself: Biomedicine, Power, and Subjectivity in the Twenty-First Century. Princeton, NJ; Oxford: Princeton University Press.

Thomson, R.G. et al (2007) A patient decision aid to support shared decision-making on anti-thrombotic treatment of patients with atrial fibrillation: Randomised controlled trial. Quality \& Safety in Health Care 16(3): 216-223. 
cc (i) This work is licensed under a Creative Commons Attribution 3.0 Unported License. The images or other third party material in this article are included in the article's Creative Commons license, unless indicated otherwise in the credit line; if the material is not included under the Creative Commons license, users will need to obtain permission from the license holder to reproduce the material. To view a copy of this license, visit http://creativecommons.org/licenses/by/3.0/ 